\title{
Evaluación de la producción quirúrgica de residentes de Cirugía General luego de la implementación del cuarto año curricular. Clínica Quirúrgica 3. Hospital Maciel (2011-2015)
}

Daniel González González*, Leandro Telles†, Cecilia Chambónł, Gustavo Armand Ugón†, Gabriela Wagnerł, Pablo Valsangiácomo§

\section{Resumen}

La formación de cirujanos generales es responsabilidad de las clínicas quirúrgicas de la Facultad de Medicina. Su programa de formación de tres años presentaba carencias en cuanto al volumen y complejidad de la cirugía que cada residente realizaba.

Objetivo: presentar los resultados de la producción quirúrgica de los residentes de Cirugía General luego de la implementación del cuarto año curricular del posgrado en la Clínica Quirúrgica 3 del Hospital Maciel y compararlos con los del período previo cuya duración era de tres años.

Material y método: estudio observacional, descriptivo y retrospectivo de la producción quirúrgica de los residentes de la Clínica Quirúrgica 3 que cursaron en el período 2011-2015. Las variables consideradas fueron: número total de cirugías realizadas por todos los residentes, promedio de cirugías totales y por año por residente, número máximo y mínimo de cirugías realizadas por un residente, porcentaje total de cirugías de coordinación y urgencia, y promedio de cirugías por residente de acuerdo a la categorización en altas, mayores, corrientes y menores. Los datos se extrajeron del sistema de descripciones operatorias de la Administración de los Servicios de Salud del Estado, Hospital Maciel.

Conclusiones: el incremento de un año en la duración de la residencia de Cirugía General determinó un aumento en el número y complejidad de la cirugía realizada por los residentes en la Clínica Quirúrgica 3 del Hospital Maciel, así como un incremento de las cirugías de coordinación en relación con las de urgencia y emergencia.

Palabras clave: Educación médica

Cirugía general

Aprendizaje

Procedimientos quirúrgicos operativos

Internado y residencia

Key words: $\quad$ Education, medical

Learning

General surgery

Surgical procedures, operative

Internship and residency

\footnotetext{
* Prof. Titular de Clínica Quirúrgica 3.

† Prof. Adjunto de Clínica Quirúrgica 3.

¥ Asistente de Clínica Quirúrgica 3.

§ Prof. Agregado de Clínica Quirúrgica 3.

Clínica Quirúrgica 3. Facultad de Medicina. Universidad de la República. Hospital Maciel.

Correspondencia: Daniel González: Atanasio Sierra 3653. Florida, Uruguay. Correo electrónico: danielalfredogg@gmail.com

Los autores declaran no tener conflicto de intereses.

Recibido: 20/5/19

Aprobado: 9/9/19
} 


\section{Introducción}

La formación de especialistas en cirugía general constituye una responsabilidad de las clínicas quirúrgicas de la Facultad de Medicina de la Universidad de la República. Su objetivo es lograr cirujanos con capacidad de manejar integralmente y con el mayor nivel profesional a personas con enfermedades quirúrgicas prevalentes.

Históricamente ha sido una preocupación en Uruguay la producción quirúrgica de los cirujanos en formación, tanto en lo referente al número de cirugías como a su nivel de complejidad.

Autores nacionales ${ }^{(1-3)}$ han comunicado carencias en dicho aspecto de la formación y ante esa realidad plantearon la necesidad de cambios. Entre los propuestos, la extensión en la duración. Esto ocurrió en 2003, cuando pasó de tres a cuatro años.

Por tal motivo, se consideró oportuno evaluar a cuatro generaciones de residentes de la Clínica Quirúrgica 3 del Hospital Maciel que finalizaron su residencia bajo el nuevo programa y si ello logró modificar los resultados de una evaluación previa bajo el programa anterior ${ }^{(3)}$.

\section{Objetivo}

Presentar los resultados de la producción quirúrgica de los residentes de Cirugía General luego de la implementación del cuarto año curricular del posgrado en la Clínica Quirúrgica 3 del Hospital Maciel y compararlos con los del período previo cuya duración era de tres años.

\section{Material y método}

Se trata de un estudio observacional, descriptivo y retrospectivo de la producción quirúrgica de los residentes de la Clínica Quirúrgica 3 que cursaron y finalizaron la residencia en el período 2011 a 2015.

Las variables consideradas fueron: número total de cirugías realizadas por todos los residentes, promedio de cirugías totales y por año por residente, número máximo y mínimo de cirugías realizadas por un residente, porcentaje total de cirugías de coordinación y urgencia, y promedio de cirugías por residente de acuerdo a la categorización de éstas en altas, mayores, corrientes y menores. Para ello, se consideró la clasificación de la Administración de los Servicios de Salud del Estado (ASSE) que se genera en forma automática en la descripción operatoria electrónica luego de seleccionar el procedimiento realizado.

Los datos se extrajeron del sistema de descripciones operatorias de ASSE, Hospital Maciel.

Las resultados de las variables fueron resumidas en porcentajes y promedios.

\section{Resultados}

Se incluyeron los últimos seis residentes que culminaron la residencia para poder comparar los resultados con el trabajo del año 2007. En el período de cuatro años realizaron un total de 2.996 cirugías, lo que determinó un promedio anual de 124,8 por cada residente. Quien operó más, realizó 596 procedimientos y el mínimo fue de 366 . El 55,3\% de las cirugías fueron realizadas en el Servicio de Urgencia y 44,7\% de coordinación en la Clínica Quirúrgica 3. Hubo un promedio por residente de $249,5 \%, 149,6 \%, 99,6 \%$ y $0,5 \%$ de cirugías altas, mayores, corrientes y menores, respectivamente.

\section{Discusión}

El análisis realizado en el año 2007 sobre la actividad quirúrgica de seis residentes de Cirugía General del Hospital Maciel en momentos en que la duración de la residencia era de tres años, concluyó que tanto el número como la complejidad de los procedimientos realizados durante su formación era insuficiente ${ }^{(3)}$.

El presente trabajo permite afirmar que hubo una mejoría de los resultados en cuanto a la producción quirúrgica de los residentes que finalizaron el programa de cuatro años de la residencia de Cirugía General en la Clínica Quirúrgica 3 del Hospital Maciel si lo comparamos tanto con el estudio llevado a cabo para residentes de los años $1999-2001^{(3)}$ y el de Franco y colaboradores ${ }^{(4)}$, momentos en que era de tres años.

Es así que hubo un incremento tanto en el promedio de cirugías realizadas por cada residente que pasó de 260 (3) y 436 (4) a 499,3 así como el número máximo y mínimo de procedimientos efectuados en cada uno de los periodos, pasando el mínimo de 223 a 366 y el máximo de 279 a 596.

También se comprobó un aumento de las cirugías realizadas de coordinación en relación con nuestra evaluación anterior, las que pasaron de $34 \%$ a $44,7 \%$, en tanto las cifras son muy similares $(46,6 \%)$ a las del trabajo de Franco y colaboradores ${ }^{(4)}$.

Si bien la complejidad de los procedimientos fue superior para el período de cuatro años de residencia, debemos aclarar que la escala utilizada para la categorización de las cirugías fue diferente. Para nuestro trabajo previo $^{(3)}$ fue la escala de la Sociedad de Cirugía del Uruguay y para el actual la de ASSE.

El programa actual de formación de posgrado de Cirugía General aprobado por la Escuela de Graduados y que extiende su duración a cinco años, establece que el residente deberá realizar entre 80 y 100 cirugías menores y corrientes en su primer año, la misma cifra de mayores en el segundo y participar como cirujano o ayudante en 70 intervenciones en el tercero y cuarto, de- 
biendo ser por lo menos el $25 \%$ de ellas complejas, y finalmente en el quinto año podrá realizar algún tiempo de una cirugía compleja. Esto nos permite afirmar que el número de pacientes que operó cada residente de la Clínica Quirúrgica 3 se encuentra dentro de las exigencias actuales del programa de posgrado de nuestro país, pero también dentro de los estándares internacionales ${ }^{(5)}$. Con respecto a la complejidad de los procedimientos, si bien el promedio de cirugía menor y corriente es menor a lo propuesto en el programa, este se ve ampliamente superado y compensado por el promedio de cirugías mayores y altas que realiza cada residente. Tal vez dicho fenómeno pueda ser explicado por la complejidad de los casos que se asisten en el Hospital Maciel, lo que posibilita que el residente participe de la resolución quirúrgica de dichos casos.

\section{Conclusiones}

El incremento de un año en la duración de la residencia de Cirugía General determinó un aumento en el número y complejidad de las cirugías realizadas por los residentes en la Clínica Quirúrgica 3 del Hospital Maciel, así como un incremento de las cirugías de coordinación en relación con las de urgencia y emergencia.

\section{Abstract}

The general surgeons training program is under the responsibility of the Surgical Clinics of the School of Medicine. The 3-year training program evidenced deficiencies in terms of the volume and complexity of the surgeries carried out by the different residents.

Objective: to present the surgical outcome of the General Surgery residents after the implementation of the fourth year in the curriculum of graduate studies of the Surgical Clinic 3 at Maciel Hospital and to compare it to that of the residents trained in the previous 3-year studies plan.

Method: observational, descriptive and retrospective study of the surgical outcome of residents of the Surgical Clinic 3 who studied in the 2011-2015 period.
Below follow the variables considered: total number of surgeries carried out by all residents, average number of surgeries per year and per resident, maximum and minimum number of surgeries by a resident, total percentage of coordination and urgent surgeries and average number of surgeries by resident according to the classification into high complexity, complex, regular and minor. Data were taken from the ASSE surgery description system - Maciel Hospital.

Conclusions: increasing one year the duration of the general surgery residence resulted in a higher number and complexity of surgeries carried out by the residents of Surgical Clinic 3 at Maciel Hospital, as well as an increase in the coordination surgeries when compared to urgency and emergency surgeries.

\section{Resumo}

A formação de cirurgiões gerais é responsabilidade das Clínicas Quirúrgicas da Facultad de Medicina. O programa de formação de 3 anos apresentava carências tanto em relação ao volume como também à complexidade da cirurgia que cada residente realizava.

Objetivo: apresentar os resultados da produção cirúrgica dos Residentes de Cirurgia Geral depois da implementação do quarto ano curricular da pós-graduação na Clínica Quirúrgica 3 do Hospital Maciel e compará-los com os do período prévio cuja duração era de 3 anos.

Material e método: estudo observacional, descritivo e retrospectivo da produção cirúrgica dos Residentes da Clínica Quirúrgica 3 que cursaram no período 2011 2015.

As variáveis estudadas foram: número total de cirurgias realizadas por todos os residentes, média de cirurgias totais e por ano por residente, número máximo e mínimo de cirurgias realizadas por residente, porcentagem total de cirurgias eletivas e de urgência e média de cirurgias por residente segundo a classificação como de grande, meio e pequeno porte e corrente. Os dados foram obtidos do sistema de descrições operatórias de ASSE Hospital Maciel.

\section{Contribución de autores}

Todos los autores participaron en igual medida en la elaboración del manuscrito.

Daniel González González, https://orcid.org/0000-0003-3916.

Cecilia Chambón, https://orcid.org/0000-0001-9388-8886.

Gustavo Armand Ugón, https://orcid.org/0000-0003-2636-0867.

Leandro Telles, https://orcid.org/0000-0003-4830-5578.

Pablo Valsangiácomo, https://orcid.org/0000-0003-2028-5552.

Gabriela Wagner, https://orcid.org/0000-0002-8526-1685. 
Conclusões: o incremento de um ano na duração da residência de cirurgia geral levou a um aumento no número e na complexidade das cirurgias realizadas pelos residentes na Clínica Quirúrgica 3 del Hospital Maciel, bem como uma aumento das cirurgias eletivas em relação às de urgência e emergência.

\section{Bibliografía}

1. Crestanello F. La formación inicial de los cirujanos generales en el Uruguay del tercer milenio y del Mercosur. Contenidos y programas. Cir Urug 2002; 72:48-57.

2. Montano D, Castro T, Curi J. Cuatro años de cirugía de urgencia. Cir Urug 1999; 69:72-7.
3. González D, Ruso L. La formación del postgrado en cirugía general. Debilidades y perspectivas desde la residencia. Rev Méd Urug 2007; 23(3):200-2.

4. Franco N, Lapiedra D, Ferraro R, Sánchez F, Curi J. Experiencia y formación del residente de cirugía en Uruguay. En: Congreso Uruguayo de Cirugía, 58 (Montevideo, 28 nov1 dic. 2007).Montevideo: Sociedad Cirugía del Uruguay, 2007.

5. García Coret M, Fuster C, Ramos M, Alberola A, Peiró F, Cañizares M, et al. Los cirujanos españoles ante la UEMS. Cir Esp 1997; 62:352. 